\title{
Estresse e comportamento alimentar
}

\author{
Stress and eating behavior \\ Strés y comportamento alimentario
}

Recebido: 02/06/2021 | Revisado: 07/06/2021 | Aceito: 08/06/2021 | Publicado: 21/06/2021

\author{
Suamily Maria Rodrigues de Matos \\ ORCID: https://orcid.org/0000-0001-9713-9428 \\ Centro Universitário Fametro, Brasil \\ E-mail: suamilym@icloud.com \\ José Carlos de Sales Ferreira \\ ORCID: https://orcid.org/0000-0002-1867-8229 \\ Centro Universitário Fametro, Brasil \\ E-mail: jcarlos.sales@gmail.com
}

\begin{abstract}
Resumo
Introdução: podemos definir como um breve conceito de estresse o entendimento como sequências de reações, que transformam a integridade física e psicológica, que aterrorizam o equilíbrio do organismo (homeostase). O estresse é um fator importante no desenvolvimento do vício e na recaída do vício e pode contribuir para um maior risco de obesidade e outras doenças metabólicas. $\mathrm{O}$ estresse incontrolável muda os padrões de alimentação e a saliência e o consumo de alimentos hiper palatáveis; com o tempo, isso poderia levar a mudanças na carga alostática e desencadear adaptações neurobiológicas que promovem um comportamento cada vez mais compulsivo. Metodologia: Optou-se pela pesquisa bibliográfica, desenvolvida a partir de material já elaborado, constituído principalmente de livros e artigos científicos. Resultados: Neste trabalho, demonstrou-se que o estresse tem sido considerado um fator de risco crítico no desenvolvimento de transtornos aditivos e recaída para comportamentos aditivos. No entanto, poucos estudos revisaram as ligações entre o estresse e a ingestão de alimentos, particularmente de alimentos hiper palatáveis ou "confortáveis" que podem ser consumidos para reduzir o estresse. Conclusão: Podemos analisar que realmente a percepção e a avaliação do estresse dependem de aspectos específicos da apresentação de estímulos externos ou internos e podem ser moderados ou mediados por traços de personalidade, estado emocional e respostas fisiológicas que, juntos, contribuem para a experiência de sofrimento.
\end{abstract}

Palavras-chave: Estresse; Metabólicas; Comportamento; Alimentação.

\begin{abstract}
Introduction: we can define as a brief concept of stress the understanding as sequences of reactions, which transform the physical and psychological integrity, which terrify the balance of the organism (homeostasis). Stress is an important factor in the development of addiction and relapse of addiction and can contribute to an increased risk of obesity and other metabolic diseases. Uncontrollable stress changes eating patterns and the overhang and consumption of hyper-palatable foods; over time, this could lead to changes in the allostatic load and trigger neurobiological adaptations that promote an increasingly compulsive behavior. Methodology: bibliographic research was chosen, developed from material already prepared, consisting mainly of books and scientific articles. Results: In this work, it was demonstrated that stress has been considered a critical risk factor in the development of addictive disorders and relapse to addictive behaviors. However, few studies have reviewed the links between stress and food intake, particularly hyper-palatable or "comfortable" foods that can be consumed to reduce stress. Conclusion: We can analyze that the perception and assessment of stress really depends on specific aspects of the presentation of external or internal stimuli and can be moderated or mediated by personality traits, emotional state and physiological responses that, together, contribute to the experience of suffering.
\end{abstract}

Keywords: Stress; Metabolic; Behavior; Food.

\section{Resumen}

Introducción: Introducción: Un concepto breve de estrés se puede definir como la comprensión de secuencias de reacciones que transforman la integridad física y psicológica, que aterrorizan el equilibrio del cuerpo (homeostasis). El estrés es un factor importante en el desarrollo de la adicción y la recaída de la adicción y puede contribuir a un mayor riesgo de obesidad y otras enfermedades metabólicas. El estrés incontrolable cambia los patrones de alimentación y la prominencia y el consumo de alimentos muy apetecibles; con el tiempo, esto podría conducir a cambios en la carga alostática y desencadenar adaptaciones neurobiológicas que promuevan un comportamiento cada vez más compulsivo. Metodología: Se optó por la investigación bibliográfica, desarrollada a partir de material ya elaborado, compuesto principalmente por libros y artículos científicos. Resultados: En este trabajo se demostró que el estrés ha sido considerado un factor de riesgo crítico en el desarrollo de trastornos adictivos y recaída en conductas 
adictivas. Sin embargo, pocos estudios han revisado los vínculos entre el estrés y la ingesta de alimentos, en particular los alimentos muy apetecibles o "cómodos" que se pueden consumir para reducir el estrés. Conclusión: Podemos analizar que la percepción y valoración del estrés realmente depende de aspectos específicos de la presentación de estímulos externos o internos y puede estar moderada o mediada por rasgos de personalidad, estado emocional y respuestas fisiológicas que, en conjunto, contribuyen a la vivencia del sufrimiento.

Palabras clave: Estrés; Metabólico; Comportamiento; Alimentación.

\section{Introdução}

Há muita verdade por trás da frase "comer estressado". O estresse, os hormônios que ele libera e os efeitos dos "alimentos reconfortantes" ricos em gordura e açucarados levam as pessoas a comer demais. No curto prazo, o estresse pode diminuir o apetite (Borinei et al., 2015).

A maioria dos humanos experimenta comportamentos alimentares alterados sob estresse, com aproximadamente $40 \%$ comendo mais e $40 \%$ comendo menos do que o normal. Além disso, sob estresse, a maioria das pessoas relata aumento na ingestão de alimentos altamente palatáveis, independente de hiperfagia ou hipofagia (Borges et al, 2017).

O sistema nervoso envia mensagens para as glândulas supra-renais sobre os rins para bombear o hormônio epinefrina (também conhecido como adrenalina). A epinefrina ajuda a desencadear a resposta de luta ou fuga do corpo, um estado fisiológico acelerado que temporariamente suspende a alimentação (Bittencourt, 2015).

A alimentação alterada também é um achado frequente em indivíduos com transtorno depressivo maior, sendo o subtipo "atípico" inclusive a hiperfagia como possível característica distintiva. Em um estudo, $46 \%$ dos participantes do estudo que preencheram os critérios para transtorno depressivo, a maior possuía características atípicas e relataram um aumento no apetite. Dos demais pacientes deprimidos sem características atípicas, $18 \%$ relataram aumento do apetite, enquanto 50\% relataram diminuição do apetite (Belém et al., 2019).

Os comportamentos alimentares complexos associados e / ou estimulados pelo estresse e pela depressão maior, sem dúvida, contribuem para o aumento do número de indivíduos com sobrepeso e obesos que experimentam ou já experimentaram estresse e depressão. A atual proliferação da obesidade crescente no mundo desenvolvido está chamando cada vez mais atenção, especialmente porque a obesidade visceral (ou central) é um fator-chave no desenvolvimento da síndrome metabólica (Catão et al., 2017).

Foi demonstrado que o aumento dos índices de estresse coincide com o aumento prevalência de obesidade e síndrome metabólica. O estresse é indicado pelo aumento da atividade do eixo hipotálamo-hipófise-adrenal e é representado por níveis elevados de cortisol plasmático (Camargo et al., 2016).

O comportamento alimentar que pode ser influenciado pelo estresse é dividido em dois componentes: o primeiro componente é a alimentação homeostática, que se relaciona à fome e saciedade e, finalmente, ao equilíbrio energético e ao peso corporal estável. O segundo componente é a alimentação não homeostática, que é influenciada pela recompensa alimentar, e é observada como comer na ausência de fome (Campos et al., 2020).

Isso sugere que o estresse interfere nas vias não homeostáticas que estão envolvidas na regulação da ingestão alimentar, como sinalização de recompensa alimentar, resultando em uma mudança de escolha alimentar para alimentos com alto teor de gordura e carboidratos. Tomados em conjunto, hipostenizamos que o estresse diminui significativamente a recompensa alimentar, levando a uma alimentação não homeostática na ausência de fome, com escolhas alimentares que resultam em maior ingestão de energia (Campos, 2016). 


\section{Metodologia}

Quanto à técnica de pesquisa, optou-se pela pesquisa bibliográfica, desenvolvida a partir de material já elaborado, constituído principalmente de livros e artigos científicos. Quase em todos os estudos é exigido algum tipo de trabalho desta natureza. Trata-se de uma pesquisa descritiva e exploratória, que segue os pressupostos científicos fazendo uso da revisão bibliográfica.

Segundo Acevedo e Nohara (2017) a pesquisa exploratória proporciona uma maior compreensão do fenômeno que está sendo investigado. Sua principal função é a explicação sistemática das coisas (fatos) que ocorrem no contexto social e que geralmente se relacionam com uma multiplicidade de variáveis. Já a pesquisa descritiva visa descrever o fenômeno que está sendo investigado, como as características do grupo, os elementos e características do comportamento desse grupo; descobrir e compreender as relações que envolvem o fenômeno investigado.

Para a coleta de dados foram usados como descritores as palavras-chave: Estresse; Educação alimentar; Distúrbios emocional; obesidade e hipertensão em bases online como Scielo (Scientific Electronic Library Online), MedLine, (Medical Literature Analysis and Retrieval System Online) Biblioteca virtual em Saúde (BVS) e Lilacs, (Literatura Latino-americana e do Caribe em Ciências da Saúde).

Para a análise de dados será feito um levantamento bibliográfico a partir de referências teórica dos últimos dez anos, entre 2012 e 2020 sobre estudos transversais, análise laboratorial, pesquisas aplicadas, delineadas a partir dos descritores, analisando-se os grupos de riscos mais encontrados, como: gestantes, idosos, crianças e adolescentes. Como critério de inclusão optou-se por analisar os estudos e pesquisas em inglês, espanhol e português, havendo certa prevalência de estudos em língua espanhola, procurando esclarecer a necessidade e a importância da interação das proteínas vegetais no treino de hipertrofia. Como critério de exclusão, documentos e estudos que não possuíam vínculo direto com os dois principais temas.

\section{Resultados e Discussão}

\subsection{Processo de atuação do estresse}

Comer bem ajuda a manter a saúde e a atividade, melhorando assim o prazer de viver. Boas dietas e hábitos alimentares são fundamentais para o crescimento e desenvolvimento adequados e para a prevenção de doenças. Vários problemas de saúde diferentes são causados por dietas e nutrição inadequadas (Costa, 2013).

Muitos desses problemas prejudicam as pessoas por toda a vida; alguns deles levam à morte. Comer bem para uma boa saúde requer um conhecimento básico dos alimentos e dos nutrientes que eles fornecem e uma compreensão das necessidades nutricionais ao longo das várias fases da vida. Atitudes e comportamentos em relação à alimentação são moldados por fatores multitudinais, incluindo fatores psicológicos e estresse (Dalmazo et al, 2019).

O estresse é um dos fatores que podem influenciar os comportamentos e a saúde, especialmente quando um indivíduo enfrenta desafios que ultrapassam suas habilidades de enfrentamento. estresse pode ser definido como "a resposta generalizada e não específica do corpo a qualquer fator que oprime, ou ameace sobrecarregar, as habilidades compensatórias do corpo para manter a homeostase (Ferreira, 2018).

O estresse psicológico, um desafio percebido ao bem-estar, é uma parte indispensável e influente da vida. Embora os fatores causadores de estresse variem muito de pessoa para pessoa e entre os sexos, as reações fisiológicas do corpo humano contra o estresse são bastante semelhantes entre si. Reações fisiológicas como pressão alta, úlcera, enxaqueca, transpiração e respiração rápida causam mudanças psicológicas como recessão, ansiedade, preocupação, aceitação e depressão. Aprender como lidar com o estresse pode fornecer a capacidade de um indivíduo de apresentar reações adequadas e suficientes contra o estresse extremo e prolongado (Ferreira, 2019). 
O estresse pode aumentar o desejo de comer de duas maneiras, diretamente ou por meio de suas propriedades que provocam a excitação. Quando os indivíduos experimentam eventos estressantes, é mais provável que desenvolvam atitudes e comportamentos alimentares desordenados. A adolescência é um período crítico da vida, tanto para o peso corporal quanto para o estresse psicológico. Estudos mostram que os adolescentes tendem a desenvolver uma imagem corporal negativa, comportamentos alimentares perturbados e altos níveis de estresse (Freitas et al., 2015).

Os hábitos alimentares desempenham um papel importante no enfrentamento do estresse, bem como dos colapsos neurológicos e psiquiátricos. Afirma-se que alguns alimentos levam a aumentar as reações de estresse, tornando os indivíduos muito mais sensíveis ao estresse. Observa-se que mulheres e pessoas com moderação consomem mais calorias e gordura sob estresse e mudam suas escolhas alimentares de alimentos do tipo refeição, como carne e vegetais, para alimentos do tipo lanche. Em contraste, os homens e os comedores desenfreados mostram pouca diferença ou uma redução na ingestão de alimentos sob estresse (França et al., 2012).

É difícil para as pessoas mudarem seus hábitos alimentares. As maneiras mais eficazes de lidar com o estresse são comer em pequenas quantidades, de maneira mais lenta e frequente, evitando alimentos ricos em gordura e açúcar, cafeína e sal e não pular refeições. Além disso, o sono regular e a prática de exercícios têm grandes benefícios para lidar com o estresse e os comportamentos alimentares desordenados (Fleig et al., 2018)

Aqueles que se exercitam regularmente e têm nutrição adequada e dietas saudáveis bem balanceadas apresentam menor frequência cardíaca e pressão arterial como uma reação em situações geradoras de estresse do que aqueles que se exercitam menos e têm uma dieta desequilibrada. Assim, fazer exercícios e ter uma alimentação adequada e balanceada é parte imprescindível do treinamento de relaxamento (Lamas et al., 2017).

\subsection{A importância de uma dieta saudável}

A importância de uma dieta saudável é amplamente aceita. Mais especificamente, as diretrizes dietéticas são formuladas na prevenção da obesidade com foco na alta ingestão de frutas e vegetais e na baixa ingestão de alimentos com alto teor calórico, como os ricos em gordura e açúcar (Lourenço, 2016).

Uma dieta geral saudável consiste em uma composição alimentar e nutritiva balanceada, bem como em um comportamento alimentar equilibrado. Um comportamento alimentar equilibrado consiste em comer com fome, em momentos regulares para permitir o crescimento fisiológico e o gasto de energia. No entanto, uma tendência de comer na ausência de fome e lanches intermitentes é cada vez mais observada no padrão alimentar na sociedade ocidental (Mayer et al., 2020).

\subsection{Tipos de estresse}

Estresse agudo: se o estressor for mais temporário e imediato, o efeito é denominado estresse agudo. $\mathrm{O}$ corpo percebe um estressor agudo (por exemplo, lidar com um congestionamento) como um desafio que o ser humano é capaz de enfrentar (Mazer et al., 2017).

Estresse crônico: se o estressor representa um aborrecimento "contínuo", medo ou uma questão avassaladora na vida de uma pessoa, o efeito é denominado estresse crônico. O estresse crônico pode parecer incontrolável (Malagris, 2019).

A resposta do corpo ao estresse agudo: Durante os períodos de estresse agudo, a parte medular do cérebro sinaliza a liberação de vários "hormônios do estresse", incluindo epinefrina (outro nome para adrenalina) e norepinefrina (outro nome para noradrenalina) das glândulas suprarrenais. Esses hormônios desencadeiam mecanismos fisiológicos de "luta ou fuga", que incluem aumentos na frequência cardíaca, frequência respiratória, quebra de gordura e carboidratos e pressão arterial (Meireles, 2016). 
Simultaneamente, o corpo retarda outros processos fisiológicos, como o fluxo sanguíneo para o sistema digestivo, o apetite e a ingestão de alimentos. $\mathrm{O}$ corpo está se preparando com a energia imediata, reflexos e força muscular de que pode precisar para agir em resposta ao estressor. Esta é uma resposta fisiológica automática a um risco real ou percebido que põe em perigo o estado atual de uma pessoa. Quando a ameaça que desencadeou a resposta foi eliminada, o corpo e a mente voltam a um estado de calma (Oliveira et al., 2019).

A resposta do corpo ao estresse crônico: Com o estresse crônico, o hipotálamo (a estação central de controle do estresse) direciona a glândula pituitária (abaixo do hipotálamo) no cérebro para enviar um hormônio de mensagem de sinalização (conhecido como hormônio adrenocorticotrófico, ou ACTH) para o córtex adrenal (a parte externa de as glândulas adrenais nos rins) (Passos et al., 2015).

O ACTH desencadeia a liberação de. Essa reação é conhecida como eixo hipotálamo-pituitária-adrenocortical (HPA) e é mais ativa em humanos durante as primeiras horas da manhã. Se o estresse crônico (real ou percebido) é de magnitude e duração suficientes, o HPA não diminui (como deveria), resultando em elevação prolongada dos níveis de cortisol (Penaforte et al., 2016).

Assim, o estresse crônico leva a aumentos diários da secreção de cortisol. O cortisol é conhecido por estimular o apetite durante os períodos intermitentes de recuperação que ocorrem quando uma pessoa está passando por estresse crônico. $\mathrm{O}$ cortisol (com a ajuda de níveis ligeiramente elevados de insulina) também demonstrou ativar a lipase da lipoproteína, a enzima que facilita a deposição de gordura (Pereira et al., 2020).

$\mathrm{Na}$ presença de níveis ligeiramente mais elevados de insulina, níveis elevados de cortisol inibem a quebra dos triglicerídeos, promovendo o armazenamento de gordura. $\mathrm{O}$ estresse crônico contribui de forma consistente para um maior acúmulo de gordura central nas mulheres. Além disso, o estresse crônico está associado a mudanças emocionais que podem incluir aumento da ansiedade, apatia e depressão. A resposta ao estresse crônico pode levar a um consumo muito maior de alimentos, conhecido como alimentação induzida pelo estresse (Pereira et al., 2019).

\subsection{Causas de estresse}

Finanças: a maioria dos estudos concorda que as finanças são uma das principais causas de estresse. Outros estão estressados por uma perda de receita ou dívida crescente de cartão de crédito.

Trabalho: O trabalho também é uma das principais causas de estresse. Trabalhos ou carreiras parecem causar estresse constante (Rosa et al., 2020).

Família: a família também é uma das principais causas de estresse. A saúde da família também é uma das principais causas de estresse. Um membro da família doente, uma lesão grave, gravidez, aborto espontâneo ou aborto causam estresse. Mudanças familiares de outros tipos também trazem estresse. Adoção, relocação e mudanças de emprego para apenas um membro da família podem causar estresse para todos (Saldanha et al., 2019).

Preocupações pessoais: as preocupações pessoais que são criadas apenas indiretamente por outras pessoas são outra das principais causas de estresse. Todo ser humano tem um desejo profundo de controle sobre sua própria vida. Para muitas pessoas, a falta de controle sobre seu próprio tempo é uma das principais causas de estresse (Souza et al., 2020).

Saúde e segurança pessoal: a maioria das pessoas acha que a saúde pessoal é a principal causa de estresse. Para alguns, o estresse está relacionado à obesidade e ao desejo de perder peso. Para outros, o estresse é um hábito pessoal básico que afeta a saúde e deve ser mudado. Por exemplo, tabagismo, abuso de álcool ou outras drogas (Silva et al., 2016).

Doenças ou lesões, sejam menos ou mais graves, podem ser uma das principais causas de estresse para muitas pessoas. A segurança pessoal também é uma das principais causas de estresse. As mulheres, mais do que os homens, tendem a se estressar com a sua própria segurança e a dos outros. 
Morte: Provavelmente a causa mais dolorosa de estresse é a morte de um ente querido ou amigo próximo (Silveira et al., 2021).

\subsection{Alimentação induzida por estresse}

A vida cotidiana exige constante restabelecimento e manutenção de um equilíbrio dinâmico em face de um ambiente em rápida mudança, é inerentemente e envolve mudanças no fluxo de energia - apetite e ingestão, armazenamento de energia e mobilização.

Em humanos, o estresse afeta a alimentação de forma bidirecional; um subgrupo, possivelmente em torno de 30\%, diminui a ingestão de alimentos e perde peso durante ou após o estresse, enquanto a maioria dos indivíduos aumenta a ingestão de alimentos durante o estresse (Silva, 2019)

Dado que as pessoas que vivem em países ocidentalizados vivem em um ambiente alimentar saboroso, com uma abundância de alimentos caloricamente densos, faz sentido que a maioria das pessoas reclame de comer mais durante o estresse, ao invés de menos. O impulso induzido pelo estresse por calorias densas é alarmante em face da crescente epidemia de obesidade (Sampaio, 2017).

A alimentação induzida pelo estresse pode ser definida como fazer com que a pessoa se sinta melhor comendo ou bebendo em resposta a uma situação estressante. Acrescente que durante os períodos de estresse crônico, as pessoas muitas vezes têm pouco tempo para preparar escolhas alimentares saudáveis e, consequentemente, tendem a escolher alimentos rápidos, que geralmente são mais calóricos (Silva et al., 2018).

Os indivíduos com excesso de peso tendem a comer mais quando expostos ao estresse crônico, enquanto indivíduos com peso normal ou abaixo do peso não. Parece que as pessoas que estão cronicamente estressadas - independentemente de comerem mais ou menos - tendem a escolher alimentos mais agradáveis ou saborosos contendo níveis mais altos de gordura e / ou açúcar (Silva, 2020)

\subsection{A resposta ao estresse}

A resposta ao estresse, que mantém a alostase, é composta por uma cascata de respostas adaptativas originadas no sistema nervoso central e também na periferia. Isso leva a mudanças fisiológicas, psicológicas e comportamentais dramáticas, mas com tempo limitado, que afetam o apetite, o metabolismo e o comportamento alimentar. A resposta ao estresse agudo inclui mudanças comportamentais, autonômicas e endocrinológicas que promovem vigilância intensificada, diminuição da libido, aumento da frequência cardíaca e pressão arterial e um redirecionamento do fluxo sanguíneo para alimentar os músculos, o coração e o cérebro (Souza et al., 2012).

Os estressores exigiram evolutivamente uma luta ou fuga imediata, de modo que a energia é desviada para o cérebro e o tecido muscular para salvar vidas. Sob tais circunstâncias, a energia gasta em atividades domésticas - como ingestão de alimentos, digestão e reprodução - seria potencialmente fatal. Portanto (Souza et al., 2019).

\subsection{Anatomia do eixo HPA}

Embora a resposta ao estresse dependa da intensidade, duração e 'tipo' de estressor, os componentes principais envolvem a ativação do eixo hipotálamo-hipófise-adrenal (HPA) e do sistema simpático adrenomedular (SAM). As estações centrais de controle da resposta ao estresse estão localizadas no hipotálamo e no tronco cerebral (Thé et al., 2019).

Os neurônios do hormônio liberador de corticotropina (CRH) do núcleo paraventricular iniciam a resposta ao estresse e constituem o principal regulador hipotalâmico do eixo hipotálamo-pituitária-adrenal (HPA). O CRH estimula a secreção de 
ACTH pela pituitária anterior. O ACTH circulante atua na zonafasciculada do córtex adrenal, onde estimula a liberação de cortisol ou corticosterona (Xavier et al., 2010).

Por sua vez, o cortisol retorna ao cérebro para interromper a secreção de cortisol. Esse ciclo de feedback negativo protege o organismo da exposição prolongada e prejudicial ao cortisol e mantém sua concentração em uma faixa operacional ampla, mas estável. O sistema simpático-adrenomedular (SAM) se origina no locus ceruleus e, juntamente com o eixo HPA, constrói os membros efetores da resposta ao estresse (Câmara et al., 2021).

\subsection{Estresse, ingestão de alimentos e obesidade.}

\subsubsection{Estresse e ingestão de alimentos}

Estão se acumulando rapidamente evidências de que o excesso de glicocorticoides desempenha um papel no desenvolvimento da obesidade por meio do aumento da ingestão de alimentos, bem como por meio da facilitação da deposição de gordura visceral.

Diferença entre gordura corporal visceral e subcutânea. A gordura corporal visceral ou central, que ocorre na área intra-abdominal do corpo, é muito diferente da gordura corporal subcutânea ou periférica, que fica logo abaixo da pele (Santos, 2021).

A gordura corporal visceral tem um fluxo sanguíneo muito maior e mais receptores de glicocorticoides. Os receptores de glicocorticoides regulam os efeitos de acúmulo de gordura do cortisol e são quatro vezes mais concentrados na gordura visceral do que na gordura subcutânea. Assim, o estresse crônico, que eleva os níveis de cortisol, resulta no acúmulo de gordura na região intra-abdominal do corpo (Saldanha et al., 2019).

\subsubsection{Pesquisa humana sobre estresse e ingestão alimentar}

Embora a complexa relação entre estresse e alimentação tenha sido reconhecida há muito tempo em humanos, os mecanismos psicobiológicos subjacentes que moldam a direção da mudança, quer se coma mais ou menos durante o estresse, são amplamente desconhecidos. Pesquisas anteriores mostraram que ser do sexo feminino, estar acima do peso ou ter uma pontuação alta na restrição alimentar são indicadores de comer mais durante o estresse (Bittencourt, 2015).

Níveis elevados de cortisol podem aumentar a ingestão calórica, como para pessoas que tomam prednisona para várias condições médicas ou tratamento de câncer. Em um estudo bem controlado, a administração de glicocorticoides aumentou significativamente a ingestão de alimentos. Presumivelmente, a alta reatividade ao estresse, que aumenta o cortisol, deve levar a uma maior ingestão de calorias, pelo menos fisicamente (Dalmazo et al., 2019).

Assim, a reatividade psicológica ao estresse pode ser uma pista para as diferenças nas características psicobiologias que explicam o estresse ao comer ou a compulsão alimentar. Em um estudo com estudantes de medicina saudáveis, comedores de estresse auto identificados tiveram cortisol urinário e insulina significativamente mais elevados durante um período estressante (exames de estudante de medicina) em comparação com um período de controle (férias de verão), e também ganharam mais peso do que aqueles que não comiam estresse, durante estresse (Campos et al., 2020).

É possível que os comedores de estresse tenham alta reatividade ao estresse subjacente, o que promove sua alimentação em excesso, embora isso não tenha sido testado diretamente. Pessoas com anorexia, bulimia e transtorno da compulsão alimentar periódica (TCAP) tendem a apresentar maior cortisol basal ou maior reatividade ao cortisol (Freitas et al., 2015). 


\subsubsection{Acúmulo de cortisol e gordura visceral}

A obesidade está associada à desregulação do eixo HPA que pode se originar do aumento do impulso para a frente, diminuição da sensibilidade à regulação do feedback negativo ou alteração da sensibilidade do tecido periférico da gordura $\mathrm{e}$ do tecido muscular esquelético aos glicocorticoides (Meireles, 2016).

Os glicocorticoides também afetam a gordura visceral por meio de seu efeito no metabolismo lipídico. Agudamente, as concentrações fisiológicas de cortisol estimulam a lipólise de todo o corpo. Na presença de insulina, as concentrações aumentadas de cortisol inibem a mobilização lipídica e favorecem o acúmulo de lipídios, seja diretamente pela estimulação da lipase lipoproteica, seja indiretamente pela inibição dos efeitos lipolíticos do hormônio do crescimento (Silva et al., 2018).

\subsubsection{Comer comedido}

Quando a alimentação é restringida, a pessoa está tentando controlar a ingestão de alimentos. Comedores reprimidos tendem a comer mais (em comparação com os comedores livres que não controlam o que comem) quando estão sob estresse crônico. Comedores contidos não apenas comem mais quando estressados; eles comem mais alimentos doces e gordurosos durante o período de alto estresse. Uma razão para essa resposta é que indivíduos que habitualmente tentam controlar seu peso regulando sua ingestão de alimentos (comedores contidos) podem eventualmente perder esse controle em situações estressantes (Ferreira, 2018).

\subsubsection{Diferença entre fome e apetite}

A fome e o apetite estão relacionados ao desejo do indivíduo de comer alimentos. Ambos desencadeiam diretamente a alimentação. A fome é comumente descrita como a necessidade psicológica e biológica de alimento. É uma condição que resulta do consumo de menos calorias do que a ingestão diária recomendada. As sensações de fome variam de um leve desconforto a verdadeiras dores de estômago. $\mathrm{O}$ apetite é o desejo físico instintivo ou a ânsia por comida. $\mathrm{O}$ apetite motiva o indivíduo a comer em um determinado horário e também o que comer. Além disso, o apetite tem um componente emocional, visto que é uma resposta aprendida intimamente associada às memórias de experiências alimentares anteriores (Lourenço, 2016).

\subsubsection{Diferença entre fome emocional e fome física}

A fome emocional surge repentinamente: atinge em um instante e parece opressora e urgente. A fome física, por outro lado, surge mais gradualmente.

A fome emocional anseia por alimentos de conforto específicos: quando está fisicamente faminto, quase tudo soa bem, incluindo coisas saudáveis como vegetais. Mas a fome emocional anseia por alimentos gordurosos ou lanches açucarados que proporcionem um ímpeto instantâneo.

A fome emocional muitas vezes leva a comer sem pensar: antes que alguém perceba, ele comeu um saco inteiro de batatas fritas ou um litro inteiro de sorvete sem realmente prestar atenção ou desfrutar totalmente. Quando alguém está comendo em resposta à fome física, ele normalmente está mais ciente do que está fazendo (Camargo et al., 2016).

A fome emocional não é satisfeita depois de saciada: a pessoa fica querendo mais e mais, muitas vezes comendo até ficar desconfortavelmente farto. A fome física, por outro lado, não precisa ser alimentada. Sentimo-nos satisfeitos quando o estômago está cheio.

A fome emocional não está localizada no estômago: em vez de uma barriga roncando ou uma dor no estômago, a pessoa sente a fome como um desejo. 
A fome emocional geralmente leva ao arrependimento, à culpa ou à vergonha: quando alguém come para satisfazer a fome física, é improvável que se sinta culpado ou envergonhado porque está simplesmente dando a seu corpo o que ele precisa. Se alguém se sente culpado depois de comer, é provável que saiba, no fundo, que não está comendo por razões nutricionais (Costa, 2013).

\subsubsection{Diferença entre homens e mulheres que sofrem de estresse crônico}

Com as mulheres, as limitações percebidas na vida diária e as tensões nas relações familiares estão associadas a um maior ganho de peso. Parece que os homens são mais propensos a lidar com o estresse crônico com outros comportamentos orais, como consumo de álcool e fumo, em vez de comer demais. No entanto, afrima-se que a falta de autoridade para tomar decisões também pode estar associada ao ganho de peso nos homens (Passos et al., 2015).

\section{Conclusão}

Embora pesquisas recentes tenham elucidado possíveis caminhos para a alimentação relacionada ao estresse, há uma necessidade considerável de tentar compreender e prevenir melhor a alimentação relacionada ao estresse e a alimentação não homeostática em geral. Apesar dos dados sugerirem propriedades potencialmente viciantes de alimentos hiper palatáveis, existe um debate sobre a existência de dependência alimentar.

Por esta e possivelmente outras razões, o vício em comida é geralmente esquecido em ambientes clínicos. Faltam programas de prevenção e tratamento em larga escala para o vício em alimentos (como aqueles para o vício em substâncias), com médicos, enfermeiras, psicólogos e outros clínicos recebendo pouco ou nenhum treinamento em vício em alimentos ou seu manejo. A identificação de biomarcadores específicos e o desenvolvimento de medidas quantificáveis para avaliar as adaptações bi comportamentais associadas ao estresse e ao vício em alimentos podem ser benéficos no desenvolvimento de intervenções de saúde pública.

O estresse é um fator importante no desenvolvimento do vício e na recaída do vício e pode contribuir para um maior risco de obesidade e outras doenças metabólicas. O estresse incontrolável muda os padrões de alimentação, a saliência e o consumo de alimentos hiper palatáveis; com o tempo, isso pode levar a mudanças na carga alostática e desencadear adaptações neurobiológicas que promovem um comportamento cada vez mais compulsivo.

A obesidade é um construto heterogêneo que, apesar de múltiplas e diversas tentativas, tem sido difícil de tratar. Uma conceituação que está ganhando atenção da mídia e da pesquisa nos últimos anos é que os alimentos, particularmente os hiper palatáveis (por exemplo, ricos em gordura e açúcar), podem possuir qualidades aditivas.

Compreender as associações e interações entre estresse, adaptações neurobiológicas e obesidade é importante no desenvolvimento de estratégias eficazes de prevenção e tratamento para a obesidade e doenças metabólicas relacionadas.

\section{Referências}

Acevedo C.R. \& Nohara J. J. (2017). Como fazer monografias: TCC, dissertações, teses.

Borinei, R. de C. C., \& Kátia, da S. W., et al. (2015). Relação entre a qualidade de vida e o estresse em acadêmicos da área da saúde. Est. Inter. Psicol. 6(1), Londrina.

Borges, K. M., Santos, F. W. dos. (2017). Síndrome do comer noturno e estados emocionais em estudantes universitários. J Hum Growth Dev. 27(3), 132139.

Bittencourt, K. F. (2015). Revisão da Literatura: Obesidade, Alimentação e Estresse. Unilasalle.

Belem, I. C., Camargo, D. A. D., et al. (2019). Diferentes comportamentos do estilo de vida de universitários do curso de educação física. Pensar en Movimiento 17(2), San José. Epub.

Catão, L. G., Tavares, R. L., et al. (2017). Técnicas da Nutrição Comportamental no Tratamento dos Transtornos Alimentares. Revista Campo Saber. 
Camargo, E. M. C., Oliveira, M. P., et al. (2016). Estresse percebido, comportamentos relacionados à saúde e condições de trabalho de professores universitários. Psicol. Argum.

Campos, L. da S., Leonel, C. F. S., et al. (2020). Relação entre estresse e obesidade: Uma revisão narrativa.

Campos, M. T. F. de S. (2016). A influência do luto no comportamento alimentar e suas implicações nas condutas nutricionais. Universidade Federal de Viçosa.

Câmara, T. A., Resende, G. C., et al. (2021). Indicadores de Comportamento Alimentar e Qualidade de Vida entre Estudantes Universitários. Amazônica Revista de Psicopedagogia, Psicologia escolar e Educação.

Costa, J. W. S. (2017). Impactos do Estresse e sua Associação com o Comportamento Alimentar dos Graduandos e, Enfermagem. Natal.

Dalmazo, A. L., Fetter, C., et al. (2019). Estresse e Consumo Alimentar em Pacientes Hipertensos. Arq. Bras. Cardiol. vol.113 no.3 São Paulo, Epub.

Ferreira, S. C. (2018). Comportamento alimentar, food craving e relação com ganho de peso e obesidade em pacientes submetidos ao transplante hepático. Belo Horizonte.

Ferreira, S. R. G. (2019). Alimentação, nutrição e saúde: avanços e conflitos da modernidade. Cienc. Cult. 62(4), São Paulo.

Freitas, E. da S. de., Canuto, R. et al. (2015). Alteração no comportamento alimentar de trabalhadores de turnos de um frigorífico do sul do Brasil. Ciênc. saúde coletiva, 20(8)

França, C. L., Biaginni, M., et al. (2012). Contribuições da psicologia e da nutrição para a mudança do comportamento alimentar. Estudos de Psicologia, $17(2)$.

Fleig, R., Nascimento, I. B. do, et al. (2018). Estresse no Ambiente Ocupacional: Fundamentos Sobre Causas e Consequências para Síndromes Metabólicas. Interfaces Científicas - Saúde e Ambiente, Aracaju, 6(2), 73 - 84

Lamas, I. \& Cadete, M. M. (2017). DO Desejo à Ação. Fatores que interferem na abordagem nutricional para mudança de hábito alimentar. Rev enferm UFPE on line., Recife.

Lourenço, A. S. de A. (2016). Ingestão de Alimentos como Mecanismo de Regulação da Ansiedade. Lisboa.

Mayer, G., Zagrobelny, A. (2020). A influência do estresse no comportamento alimentar em profissionais da área de enfermagem: uma revisão integrativa. CESCAGE.

Mazer, L. C., Rodrigues, A. A., et al. (2017). Percepção de Stress e Comportamento Alimentar de Estudantes de Psicologia.

Malagris, L. E. N. (2019). Stress, resiliência e apoio social em indivíduos com hipertensão e diabetes mellitus. Rev. psicol. 28(1) Santiago.

Meireles, M. R. (2016). Influência de condições de estresse no comportamento alimentar.

Oliveira, M. M. A., Marinho, M. F., et al. (2019) A Influência do Estresse no Comportamento Alimentar dos Estudantes Universitários: Uma Revisão DE literatura. Fortaleza.

Passos, D. R. dos., Gigante, D. P., et al. (2015). Comportamento alimentar infantil: comparação entre crianças sem e com excesso de peso em uma escola do município de Pelotas, RS. Pelotas, RS, Brasil.

Penaforte, F. R. de O., Matta, N. C., Japur, C. C., et al. (2016). Associação entre estresse e comportamento alimentar em estudantes universitários. Demetra.

Pereira, M. D., et al. (2020). A Pandemia de COVID-19, o isolamento social, consequências na saúde mental e estratégias de enfrentamento: uma revisão integrativa. Revista Research.

Pereira, T. da C., et al. (2019). Estado Emocional e Comportamento Alimentar de Universitárias de Uma Instituição de Ensino Particular. Revista Ciência.

Rosa, C. A. S., et al. (2020). Correlação entre Ansiedade ou Estresse e Comportamento Alimentar em Estudantes Universitários. Maceió/AL.

Santos, D. R. C. dos. (2021). Comportamento Alimentar x Depressão: Uma revisão de Literatura. Governador Mangabeira -BA.

Sadanha, A. B. R., et al. (2019). Aspectos Relacionados à Qualidade de Vida de Estudantes durante a jornada acadêmica: uma revisão de literatura. FASB.

Souza, M. P. G., et al. (2020). Comportamento Alimentar e Fatores Associados em Servidores: Contribuições Para a Saúde Coletiva. Rev. Aten. Saúde, São Caetano do Sul, 18(63), 99-109.

Silva, J. K., Prado, S. D., et al. (2016). Alimentação e Nutrição: do que estamos falando? Physis Revista de Saúde Coletiva, Rio de Janeiro, $26(4), 1103-1123$.

Silveira, M. R. \& Costa, A. A. da. (2021). A Influência do Estresse no Comportamento Alimentar. IMEA.

Silva, D. S. (2019). A influência do estresse no comportamento alimentar e no estado nutricional de estudantes de nutrição. Unisul.

Sampaio, C. (2017). Estresse, Comportamento Alimentar e Excesso de Peso. congresso brasileiro de psicoterapias corporais, XXII.

Silva, R. M., Goulart C. T., et al. (2018). Evolução histórica do conceito de estresse. Rev. Cient. Sena Aires. 7(2), 148-56.

Silva, D. R. P. da. (2020). Distanciamento social, sentimento de tristeza e estilos de vida da população brasileira durante a pandemia de COVID-19. 
Research, Society and Development, v. 10, n. 7, e26210716726, 2021

(CC BY 4.0) | ISSN 2525-3409 | DOI: http://dx.doi.org/10.33448/rsd-v10i7.16726

Souza, A. S., et al. (2012). O Comportamento Alimentar e os Distúrbios psicológicos.

Souza, D. T. B. de., et al. (2019). Ansiedade e Alimentação: Uma Análise Interrelacional. Faculdades Integradas de Patos - FIP.

Thé, M. B. S., \& Corrêa, M. A. (2019). Consequências do Estresse no Estado Nutricional dos Manipuladores de Unidades de Alimentação e Nutrição. Centro Universitário de Várzea Grande.

Xavier, M. de F., et al. (2020). Avaliação do Estresse, Estilo Alimentar e Qualidade de Vida em Praticantes de Atividade Física e Sedentários. CPAQV Journal. 